\title{
Assessment of the optic nerve and its arterial supply by ultrasound imaging in multiple sclerosis patients
}

\author{
Manal Mahmoud Al Kattan', Amira Ahmed Labib², Rania Shehata Ismail ${ }^{1 *}$, Alshaimaa M. Aboul fotouh ${ }^{1}$ and \\ Emad El Din Mostafa Mohammed ${ }^{1}$
}

\begin{abstract}
Background: Transorbital sonography (TOS) has emerged as a promising assessment tool of the optic nerve and orbital arterial supply in multiple sclerosis (MS) patients.

Objective: To evaluate optic nerve diameter (OND), optic nerve sheath diameter (ONSD), and orbital hemodynamics using TOS in MS patients.

Methods: Forty MS patients and 28 matched healthy controls were included. Thirty-three eyes with previous optic neuritis $(\mathrm{ON})$ and 47 eyes without previous ON of MS patients were examined. All subjects were submitted to assessment of OND, ONSD, and parameters of orbital hemodynamics using orbital ultrasound.

Results: OND and ONSD were smaller in MS eyes with previous ON than in controls. MS eyes with and without previous ON had statistically significant higher peak systolic and mean velocity of posterior ciliary arteries than the control eyes. Orbital blood flow velocities were negatively correlated with the duration of disease and Expanded Disability Status Scale (EDSS). A statistically significant decrease in blood flow velocities of the central retinal artery was detected in secondary progressive MS (SPMS) patients than in relapsing-remitting MS patients (RRMS).

Conclusion: TOS can be used as a feasible tool to detect optic atrophy in MS patients. MS patients may have abnormal retrobulbar hemodynamics compared to healthy controls.
\end{abstract}

Keywords: Transorbital sonography, Optic nerve, Multiple sclerosis

\section{Introduction}

Optic neuritis $(\mathrm{ON})$ is an immune-mediated acute inflammatory disorder of the optic nerve and represents a common initial manifestation of multiple sclerosis (MS) [1]. The optic nerve is most commonly assessed by ophthalmoscopy and magnetic resonance imaging (MRI), but recently, ultrasound has emerged as a promising additional assessment tool. Being filled with aqueous fluid, the eye is well appropriate for ultrasound imaging. The role of optic nerve ultrasound in the detection of increased ICP has been widely investigated in brain injuries, intracranial hemorrhage, hydrocephalus, brain tumors, and idiopathic intracranial hypertension. However, few studies evaluated

\footnotetext{
* Correspondence: raniashehata.neuro@yahoo.com

'Department of Neurology, Faculty of Medicine, Cairo University, Giza, Egypt

Full list of author information is available at the end of the article
}

its use to detect optic nerve changes in multiple sclerosis patients [2].

Color Doppler imaging (CDI) is a non-invasive imaging method that provides information about changes in the hemodynamics of orbital vessels in various disorders, such as MS, Behcet's syndrome, arterial hypertension, glaucoma, and uveitis [3].

The aim of this work is to evaluate optic nerve diameter (OND) and optic nerve diameter sheath (ONSD) and parameters of orbital hemodynamics using orbital ultrasound in eyes with previous optic neuritis, to compare these values with healthy control eyes and eyes without previous optic neuritis in MS patients, and also to correlate these values with the disease duration and disability. 


\section{Materials and methods}

In this case-control study, 40 patients with clinically definite MS were recruited from Kasr Al Ainy Multiple Sclerosis Unit (KAMSU), Cairo University, between November 2015 and February 2017 according to the revised McDonald's criteria 2017 [4]. The aim and procedures of the study were explained to every participant, and an informed consent was obtained before being enrolled in the study.

The patients were divided into two groups: The patient group (A) included $40 \mathrm{MS}$ patients with a total number of 80 eyes, whereas the control group (B) included 28 healthy subjects matched in age and gender to MS patients with a total number of 56 healthy control eyes. All patients were submitted to the following: general, ophthalmological, and neurological evaluation; assessment of disability using the Expanded Disability Status Scale (EDSS); magnetic resonance imaging (MRI) of the brain (1.5 T IGC F2000 Magnet 2016 USA Phillips Intera ${ }^{\circ}$ scanner); pattern-reversal visual evoked potentials (P-VEP) (Neuropack M1 MEB-9200); transorbital sonography (TOS); and color Doppler imaging using Philips IU22 with software version 2.0.13 USA 2012 with linear transducer probe L 9-3; ONSD and OND were assessed $3 \mathrm{~mm}$ behind the papilla; and the ophthalmic artery (OA) was examined approximately 30-35 mm behind the globe. Posterior ciliary arteries (PCAs) were examined temporal to the optic nerve approximately within $5 \mathrm{~mm}$ behind the globe, and the results were averaged. The central retinal artery (CRA) is examined within $5 \mathrm{~mm}$ of the retrolaminar portion of the optic nerve [5].

\section{Statistical analysis}

The collected data were coded, tabulated, and statistically analyzed using IBM SPSS statistics (Statistical Package for Social Sciences) software version 22.0, IBM Corp. Descriptive statistics were done for quantitative parametric data as mean \pm SD and for qualitative data as number and percentage. Inferential analyses were done for quantitative variables using independent $t$ test in cases of two independent groups with parametric data and paired $t$ test in cases of two dependent groups with parametric data and ANOVA test for more than two independent groups with parametric data; then, post hoc test was used for pair-wise comparison based on Kruskal-Wallis distribution. Inferential analyses for qualitative data were done using the chi-square test for differences between proportions and Fisher exact test for variables with small expected numbers. Correlations between quantitative variables were done using Spearman correlation coefficient. A multi-variate linear regression model was used. The level of significance was considered as follows: $\rho$ value $<0.05$ is significant, and $\rho$ value $\geq 0.05$ is non-significant.

\section{Results}

\section{Demographic and clinical data}

The mean age of MS patients was $33.75 \pm 9.43$ years while the mean age of controls was $33.25 \pm 6.12$ years; out of 40 patients with MS, 17 patients (42.5\%) were males and 23 patients $(57.5 \%)$ were females, while in the control group, 11 subjects (39.3\%) were males and 17 subjects $(60.7 \%)$ were females.

The age of onset of the disease ranged from 12 to 43 years with a mean age of onset $25.53 \pm 8.67$ years. The duration of the disease ranged from 1 to 27 years with a mean $8.25 \pm 6.59$ years. The duration since optic neuritis attack in patients with previous $\mathrm{ON}$ ranged from 1 to 15 years with mean $4.3 \pm 3.36$ years.

EDSS of patients ranged from 1.5 to 7 with a mean EDSS of $4.11 \pm 1.89$.

Among the $40 \mathrm{MS}$ patients, 12 patients (30.0\%) were diagnosed to have secondary progressive MS (SPMS) while 28 patients $(70.0 \%)$ were diagnosed to have relapsing-remitting MS (RRMS).

\section{Frequency of ON}

Twenty-one MS patients had previous $\mathrm{ON}$ at least 3 months before the examination. Of these patients, 3 patients had $\mathrm{ON}$ in the right eye, 6 patients had $\mathrm{ON}$ in the left eye, and 12 patients had $\mathrm{ON}$ in both eyes, with a total number of 33 affected eyes. Nineteen MS patients had no previous $\mathrm{ON}$ with a total number of 47 unaffected eyes included in the study.

\section{Comparative results}

Eyes of MS patients had significant lower OND and ONSD than control eyes ( $p$ value $=0.032,0.023$ ) respectively. Moreover, there was a statistically significant difference in OND and ONSD between the MS eyes with previous $\mathrm{ON}$, MS eyes without previous $\mathrm{ON}$, and healthy control eyes ( $p$ value $=0.029,0.019$ ) respectively. By post hoc analysis, there was a statistically significant decrease in OND and ONSD in the group of MS eyes with previous $\mathrm{ON}$ than the control group ( $p$ value $0.024,0.015$ ) respectively. However, no statistically significant difference in OND and ONSD between MS eyes with previous ON and MS eyes without previous $\mathrm{ON}(p$ value $=0.33,0.64)$ respectively. Also, no statistically significant difference was found between MS eyes without previous optic neuritis and control eyes in both OND and ONSD ( $p$ value $=0.74,0.648$ ) respectively.

Regarding assessment of orbital hemodynamics in the studied groups using color Doppler imaging, there was a statistically significant difference in peak systolic velocity (PSV), mean velocity (MV), and end-diastolic velocity (EDV) of the posterior ciliary artery (PCA) between MS eyes with, without previous $\mathrm{ON}$, and healthy controls ( $p$ value $=0.002,0.002$, and 0.022) respectively. By post hoc 
analysis, MS eyes with and without previous $\mathrm{ON}$ had statistically significant higher PSV and MV of PCA than healthy controls ( $p$ value $<0.05)$ (Fig. 1). Moreover, MS eyes without previous $\mathrm{ON}$ had a statistically significant higher EDV than the control group ( $p$ value $=0.018$ ). However, there was no statistically significant difference between MS eyes with and without previous $\mathrm{ON}$ and the control group in PSV, MV, EDV, RI (resistivity index), and PI (pulsatility index) of the central retinal artery( CRA) and ophthalmic artery (OA). There was a statistically significant decrease in PSV, EDV, and MV of CRA in SPMS patients compared to RRMS patients. However, there was no statistically significant difference between RRMS eyes and SPMS eyes in PSV, MV, EDV, RI, and PI of the ophthalmic and posterior ciliary arteries.

\section{Correlations between ultrasound findings and clinical data in MS eyes with previous optic neuritis}

Central retinal artery PSV was negatively correlated with both EDSS and duration of disease ( $p=0.01, r=0.044 ; p=$ $0.009, r=0.44$ ) respectively (Fig. 2). Ophthalmic artery PSV and MV were also negatively correlated with the duration of the disease $(p=0.01, r=-0.044 ; p=0.014, r=$ 0.42 ) respectively (Fig. 3). However, no correlation was found between OND and ONSD with the age of onset of the disease, duration of the disease, or EDSS of the patients.

The multivariate linear regression analysis was used to detect the predictors for PSV of CRA in MS eyes with previous optic neuritis. The duration of the disease and disease severity assessed by EDSS were used as independent predictors in the model. We found that the only predictor for PSV of CRA was EDSS (beta $=-0.41, p$ value $=0.018)$.

\section{Discussion}

In this study, the optic nerve was examined in $40 \mathrm{MS}$ patients using TOS. We assessed also the arterial supply of optic nerve using color Doppler imaging.

The current study revealed that MS patients had smaller OND and ONSD than healthy controls. Moreover, MS eyes with previous ON had smaller OND and ONSD than eyes of healthy controls. However, we could not find a statistically significant difference in OND and ONSD between MS eyes without previous ON and eyes of healthy controls. This was in accordance with Carraro and colleagues [6]. Similar to Candeliere Merlicco and colleagues [7], we did not find statistically significant difference in OND between patients with and without previous optic neuritis. They believed that sample size might have been insufficient to reach significance and some MS patients without history of ON could have had a silent $\mathrm{ON}$.

We found no statistically significant difference in OND and ONSD between RRMS and SPMS. Also, no correlation exists between OND or ONSD and the duration of the disease or EDSS. This finding was supported by results of previous studies that found that retinal

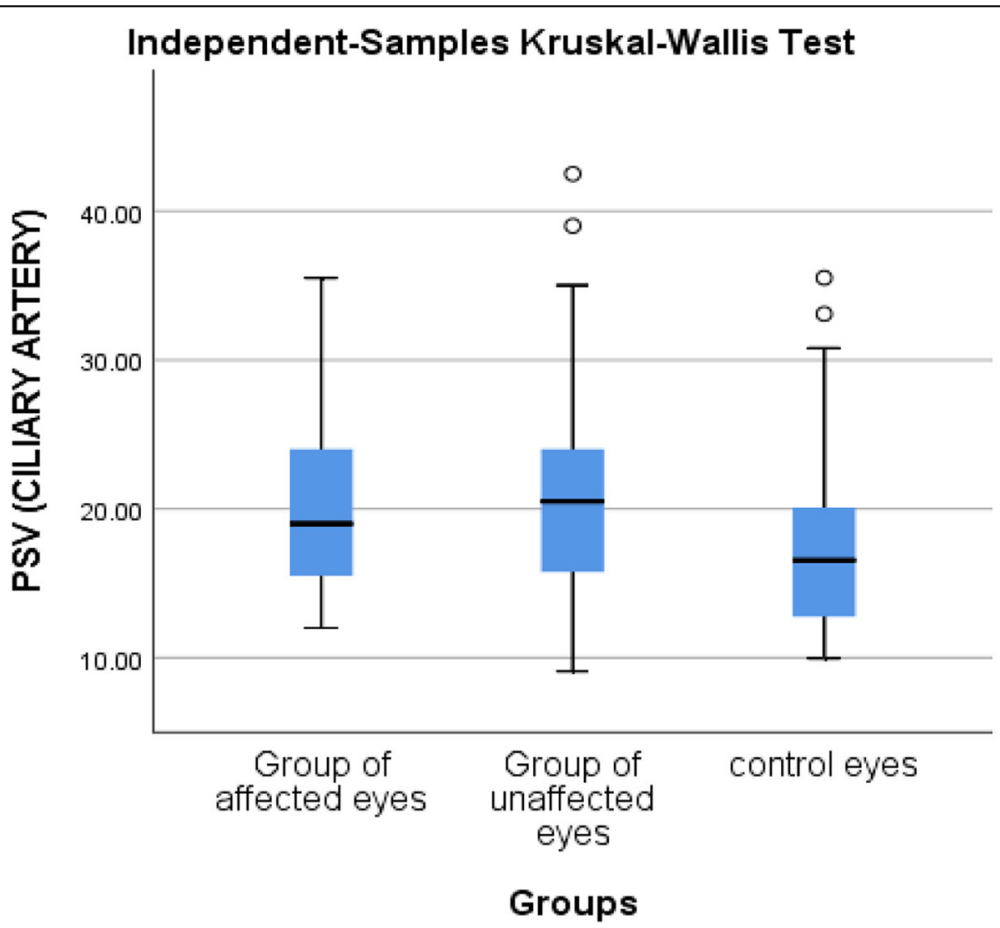

Fig. 1 PSV of the posterior ciliary artery in the studied groups. PSV peak systolic velocity 


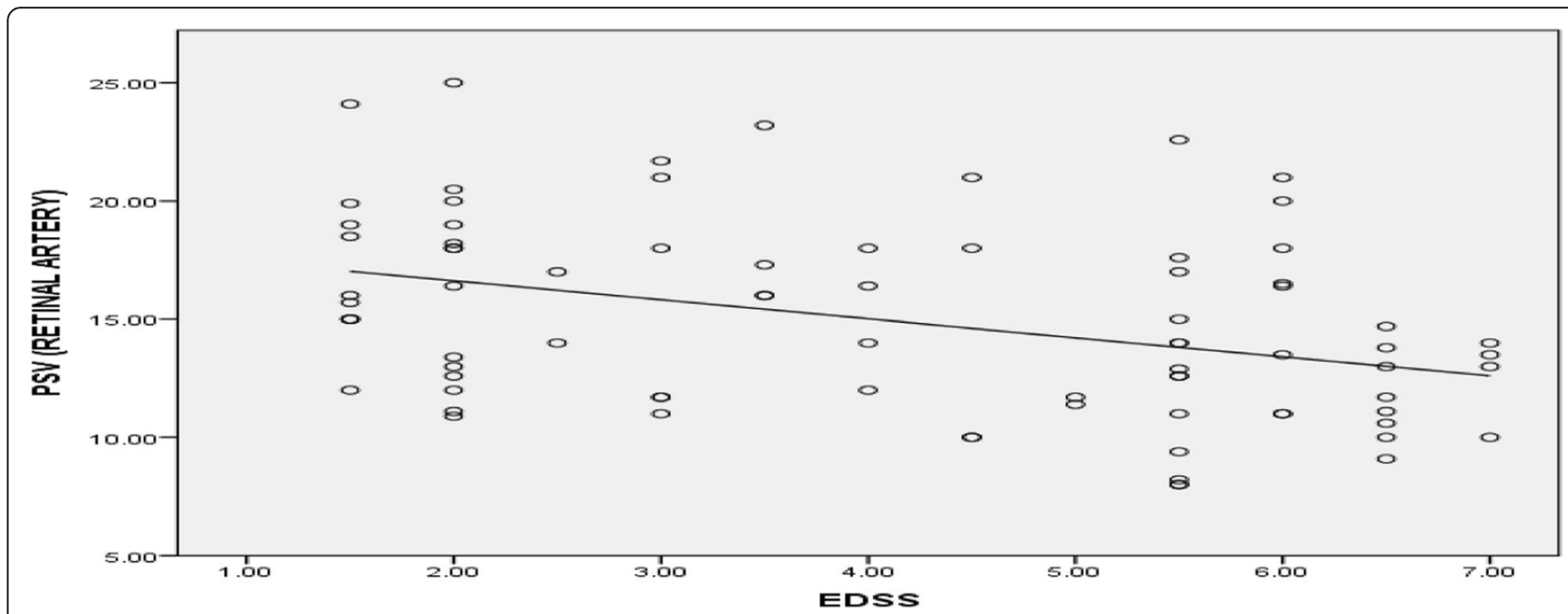

Fig. 2 Correlation between PSV of the central retinal artery and EDSS in MS patients. EDSS Expanded Disability Status Scale, MS multiple sclerosis, PSV peak systolic velocity

nerve fiber layer (RNFL) and macular thicknesses were not statistically reduced over time [8]. On the contrary, De Masi and colleagues [9] found a negative correlation between ONSD and EDSS but not disease duration, whereas Candeliere Merlicco and colleagues [7] found a negative correlation between OND and both duration of the disease and EDSS. The dissimilarity of results can be attributed to different sample size and clinical characters (type of MS, duration of the disease, and EDSS).

Vascular changes have also been described as occurring more often in MS patients [10] particularly in the eye [11]. The inflammation also involves vascular and perivascular tissue and thereby influences brain perfusion [12].

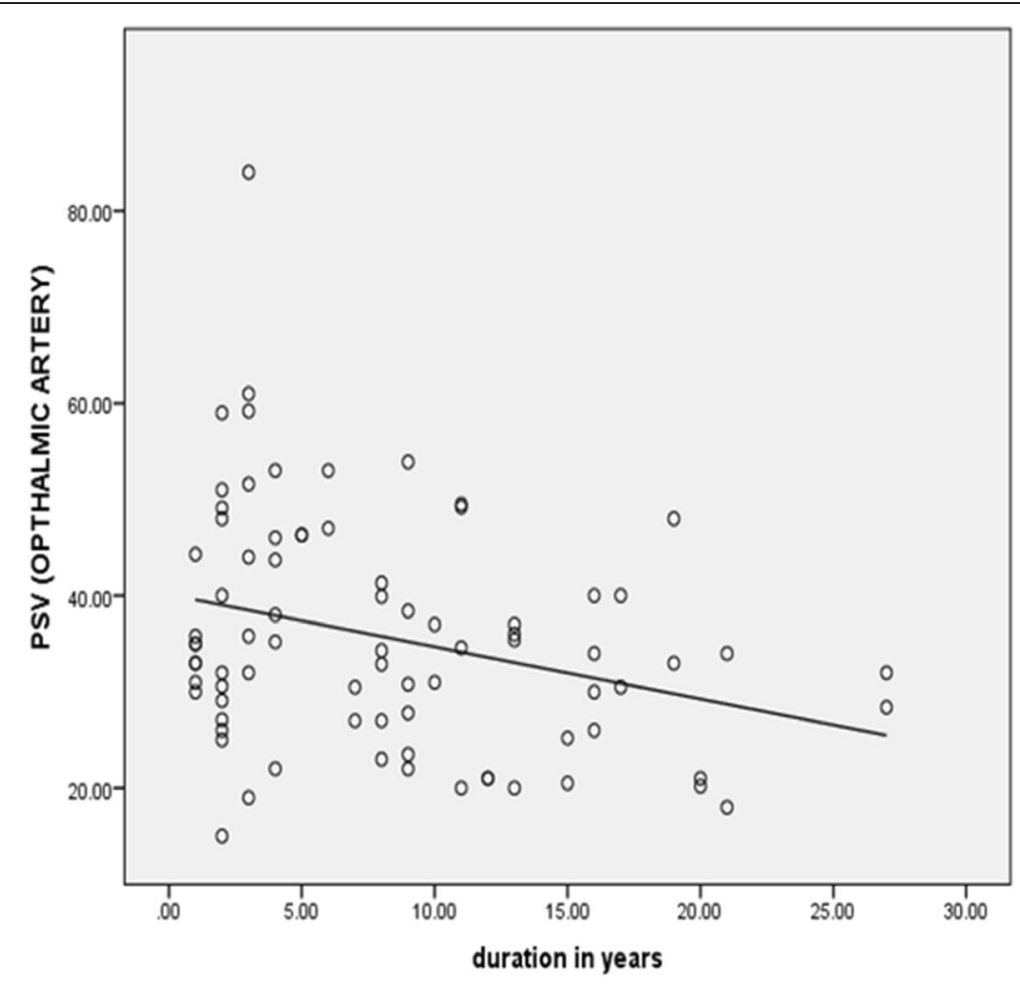

Fig. 3 Correlation between PSV of the ophthalmic artery and duration of disease in MS patients. MS multiple sclerosis, PSV peak systolic velocity 
We found that MS eyes with and without ON had higher blood flow velocities of PCAs than control eyes. This may be attributed to increased levels of endothelin1 (ET1) which might have resulted in vasospasm and vascular dysregulation [13]. This was supported by previous studies that demonstrated increased ET-1 level in CSF and blood of MS patients [14]. However, we did not find a statistically significant difference between MS eyes with and without $\mathrm{ON}$ and control eyes in hemodynamics of OA and CRA. This goes in accordance with Carraro and colleagues [6] who did not find any significant difference in any of the blood velocities, RI or PI of OA and CRA between healthy controls eyes, MS affected eyes, and MS unaffected eyes. Pache and colleagues [13] examined hemodynamics of retrobulbar arteries in 30 MS patients. They found a significant reduction in the PSV and EDV of the OA, PCAs, and CRA in MS patients compared with healthy controls. They found no significant differences of blood flow velocities between patients with and without a history of ON. Modrzejewska and colleagues [15] reported statistically significant diminishing blood flow velocity parameters in the orbital arteries of 16 patients with previous $\mathrm{ON}$. They found diminished MV in OA; PSV, MV, RI in CRA; and PSV, EDV, MV and RI in PCA in affected eyes. Akcam and colleagues [16] found no difference in blood flow velocity and mean resistivity index parameters of OA, CRA, and PCA arteries between MS patients with and without a history of optic neuritis and healthy control. This discrepancy may be explained in part by different sample size and diverse clinical characteristics (EDSS, course, and duration of the disease), the cause of $\mathrm{ON}$, and the amount of time elapsed between the acute attack and examination.

The present work revealed that patients with SPMS had diminished blood flow velocities in CRA than patients with RRMS. This may be explained by increased axonal loss in SPMS and the hypothesis that an optic nerve that is compromised by axonal loss has a lower demand for blood supply. To the best of our knowledge, this is a pioneer study comparing hemodynamics of retrobulbar vessels between RRMS and SPMS.

Moreover, we found a negative correlation between orbital hemodynamics and EDSS and duration of disease. This may be explained by increased axonal loss associated with increased EDSS and prolonged disease duration and consequently decreased metabolic demands of the optic nerve. Furthermore, we found that EDSS may be used as a predictor for PSV of CRA.

\section{Conclusion}

From all previous results, it could be concluded that TOS could be used as a feasible tool to detect optic atrophy in MS patients with previous optic neuritis. Moreover, this study suggests that MS patients have abnormal retrobulbar hemodynamics compared to normal subjects. This indicates vascular dysregulation in MS. Further studies are needed to clarify the role of the retrobulbar hemodynamics in the pathogenesis of MS and to determine whether the impaired retrobulbar hemodynamics is a primary issue and a predictor of underlying neurodegeneration in MS.

\section{Abbreviations \\ CRA: Central retinal artery; EDSS: Expanded Disability Status Scale: \\ ET1: Endothelin-1; MRI: Magnetic resonance imaging; MS: Multiple sclerosis; MV: Mean velocity; OA: Ophthalmic artery; ON: Optic neuritis; OND: Optic nerve diameter; ONSD: Optic nerve diameter sheath; PCAs: Posterior ciliary arteries; PI: Pulsatility index; PSV: Peak systolic velocity; P-VEP: Pattern-reversal visual evoked potentials; RFI: Retinal function imager; RI: Resistivity index; RNFL: Retinal nerve fiber layer; RRMS: Relapsing-remitting MS; \\ SPMS: Secondary progressive MS; TOS: Transorbital sonography; VEP: Visual evoked potentials}

\section{Acknowledgements \\ The authors acknowledge the subjects for their participation and cooperation in this study.}

\section{Authors' contributions}

MME contributed to the research idea, data acquisition, and data analysis and interpretation. AAL contributed to the research idea, data acquisition, and data analysis and interpretation and performed neurophysiological workup. RSI and AMA contributed to the research idea, data acquisition, data analysis and interpretation, and manuscript writing and review. EMM contributed to the research idea, data acquisition, and data analysis and interpretation. All authors read and approved the final manuscript.

\section{Funding}

This research received no specific grant from any funding agency in the public, commercial, or not-for-profit sectors.

\section{Availability of data and materials}

The datasets generated and/or analyzed during the current study are not publicly available due to the current Cairo University regulations and Egyptian legislation but are available from the corresponding author on reasonable request and after institutional approval.

\section{Ethics approval and consent to participate}

An informed written consent was taken from each patient. All data obtained from every patient were confidential and were not used outside the study. The patients have rights to withdraw from the study at any time without giving any reason. All the cost of the investigations was afforded by the researcher.

Our study was approved by the ethical committee of the Department of Neurology, Faculty of Medicine, Cairo University, on 5th of May 2017, but Cairo University does not provide approval reference number.

\section{Consent for publication}

Not applicable.

\section{Competing interests}

The authors declare that they have no competing interests.

\section{Author details}

${ }^{1}$ Department of Neurology, Faculty of Medicine, Cairo University, Giza, Egypt. ${ }^{2}$ Department Clinical Neurophysiology, Faculty of Medicine, Cairo University, Giza, Egypt. 
Received: 25 May 2019 Accepted: 29 August 2019

Published online: 13 September 2019

\section{References}

1. Cheng H, Laron M, Schiffman JS, Tang RA, Frishman LJ. The relationship between visual field and retinal nerve fiber layer measurements in patients with multiple sclerosis. Invest Ophthalmol Vis Sci. 2007;48(12):5798-805.

2. Tawfik EA, Walker FO, Cartwright MS. Neuromuscular ultrasound of cranial nerves. J Clin Neurol. 2015;11(2):109-21.

3. Hradílek P, Stourac P, Bar M, Zapletalová O, Školoudík D. Colour Doppler imaging evaluation of blood flow parameters in the ophthalmic artery in acute and chronic phases of optic neuritis in multiple sclerosis. Acta Ophthalmol. 2009;87(1):65-70.

4. Thompson AJ, Banwell BL, Barkhof F, Carroll WM, Coetzee T, Comi G, et al. Diagnosis of multiple sclerosis: 2017 revisions of the McDonald criteria. Lancet Neurol. 2018;17(2):162-73.

5. Walasik-Szemplińska D, Pauk-Domańska M, Sanocka U, Sudoł-Szopińska I. Doppler imaging of orbital vessels in the assessment of the activity and severity of thyroid-associated orbitopathy. J Ultrason. 2015;15(63):388.

6. Carraro N, Servillo G, Maria Sarra V, Bignamini A, Pizzolato G, Zorzon M. Optic nerve and its arterial-venous vascularization: an ultrasonologic study in multiple sclerosis patients and healthy controls. J Neuroimaging. 2014; 24(3):273-7.

7. Candeliere Merlicco A, Gabaldón Torres L, Villaverde González R, Fernández Romero I, Aparicio Castro E, Lastres Arias MC. Transorbital ultrasonography for measuring optic nerve atrophy in multiple sclerosis. Acta Neurol Scand. 2018;138(5):388-93.

8. Serbecic N, Aboul-Enein F, Beutelspacher SC, Vass C, Kristoferitsch W, Lassmann H, Reitner A, Schmidt-Erfurth U. High resolution spectral domain optical coherence tomography (SD-OCT) in multiple sclerosis: the first follow up study over two years. PLoS One. 2011;6(5):e19843.

9. De Masi R, Orlando S, Conte A, Pasca S, Scarpello R, Spagnolo P, et al. Transbulbar B-mode sonography in multiple sclerosis: clinical and biological relevance. Ultrasound Med Biol. 2016;42(12):3037-42.

10. Marrie RA, Reider N, Cohen J, Stuve O, Trojano M, Cutter G, et al. A systematic review of the incidence and prevalence of cardiac cerebrovascular, and peripheral vascular disease in multiple sclerosis. Mult Scler J. 2015;21(3):318-31.

11. Jiang H, Delgado S, Tan J, Liu C, Rammohan KW, DeBuc DC, et al. Impaired retinal microcirculation in multiple sclerosis. Mult Scler J. 2016;22(14):1812-20.

12. Konieczka K, Koch S, Binggeli T, Schoetzau A, Kesselring J. Multiple sclerosis and primary vascular dysregulation (Flammer syndrome). EPMA J. 2016;7(1):13.

13. Pache M, Kaiser HJ, Akhalbedashvili N, Lienert C, Dubler B, Kappos L, et al. Extraocular blood flow and endothelin-1 plasma levels in patients with multiple sclerosis. Eur J Neurol. 2003;49(3):164-8.

14. Speciale L, Sarasella M, Ruzzante S, Caputo D, Mancuso R, Calvo MG, et al. Endothelin and nitric oxide levels in cerebrospinal fluid of patients with multiple sclerosis. J Neurovirol. 2000:6:S62-6.

15. Modrzejewska M, Karczewicz D, Wilk G. Assessment of blood flow velocity in eyeball arteries in multiple sclerosis patients with past retrobulbar optic neuritis in color Doppler ultrasonography. Klin Oczna. 2007;109(4-6):183-6.

16. Akcam HT, Capraz IY, Aktas Z, Caglayan HB, Oktar SO, Hasanreisoglu M, et al. Multiple sclerosis and optic nerve: an analysis of retinal nerve fiber layer thickness and color Doppler imaging parameters. Eye. 2014;28(10):1206.

\section{Publisher's Note}

Springer Nature remains neutral with regard to jurisdictional claims in published maps and institutional affiliations.

\section{Submit your manuscript to a SpringerOpen ${ }^{\circ}$ journal and benefit from:}

- Convenient online submission

- Rigorous peer review

- Open access: articles freely available online

- High visibility within the field

- Retaining the copyright to your article

Submit your next manuscript at $\boldsymbol{\nabla}$ springeropen.com 